\title{
LA BUENA GESTIÓN DE LA COMUNICACIÓN INTERNA UNA VENTAJA A FAVOR DE LAS EMPRESAS ANTE LOS CONFLICTOS
}

\author{
Magdalena Mut Camacho: Universitat Jaume I de Castelló (España)
}

\section{Introducción}

La Comunicación Interna es un instrumento de gestión eficaz que todo director de empresa debe saber manejar hábilmente con vistas a una mayor rentabilidad, y es cierto que es uno de los campos todavía por explotar en las empresas, por beneficiaRse de sus enormes ventajas y beneficios. En el caso que queremos analizar, partiremos de la base de que, entre sus muchas ventajas, la Comunicación Interna contribuye a facilitar el cambio, reduciendo el grado de resistencia, incrementando el nivel de compromiso y fomentando la adaptación a nuevas óituaciones; es aquí donde encontramos unos de los factores que ayudarán a la pronta solución de conflictos internos y a no perder el control entre los empleados en los momentos de crisis.

Harold Burson, fundador de Bursmn Marsteller, una de las agencias de relaciones públicas más importantes del mundo, es tajante a la hora de defender la Comunicación Interna, una de las gestiones que considera todavía por aprovechar\$en las empresas: «La comunicación con los empleados es el primer objetivo de una empresa. Si no tienes gente que entienfa y comparta tu mensaje dentro, no puedes pretender venderlo fuera ${ }^{1}$.

La Comunicación Interna no es simplemente una herramienta clave en la gestión empresarial, sino que actualmente ha de ser concebida como una estrategia importante del management empresarial. «La Comunicación Interna es el principal artífice de la transparencia en la organización, y, por lo tanto, no debe ser considerada como un mero vehículo de transmisión de la información. Ni siguiera como un buen método de gestión a seguir de vez en cuando. Hay que verla como una función inherente a cualquier tarea y es necesario concretarla y definirla con precisión»².

En España, en el año 2003, Dircom, asociación de Directivos de Comunicación, efectuó un estudio titulado Expectativas, prácticas y resultados de la Comunicación Interna en empresas e instituciones españolas ${ }^{3}$. Las principales conclusiones obtenidas fueron las siguientes:

Tan solo un $41 \%$ de las empresas dispone de un plan estructurado de Comunicación Interna, y el $21 \%$ no tiene ningún plan de estas características.

Entre los que sí disponen de un plan, la mayoría afirma que los mandos intermedios y la Alta Dirección no se implican lo suficiente en la elaboración del plan ni en las acciones de comunicación.

Casi todos los planes tienen objetivos informativos pero la creación de ocasiones de diálogo interno y el establecimiento de compromisos de comunicación en los equipos, sólo se contempla en algo más de la mitad de los planes.La práctica

\footnotetext{
$1 \quad$ www.expansionnyempleo.com. 09 de julio de 2003

2 Cervera, L.A. Comunicación total. Esic. Madrid, 2004

3 Dircom. Expectativas, prácticas y resultados de la Comunicación Interna en empresas e instituciones españolas. Estudio de opinión informada en organizaciones líderes en Comunicación Interna. Dircom. Madrid, 2003.
}

Recibido: 01/03/2006---Aceptado: 16/04/2006---Publicado: 12/11/2006 
totalidad de los altos directivos reconoce la influencia de la Comunicación Interna en el clima interno y la motivación, pero un $11 \%$ no la vincula con la productividad y el rendimiento. Un $25 \%$ percibe poca o ninguna relación entre Comunicación Inuerna y cuenta de resultados.

Por tanto, vemks como en España ya existe una cierta cultura de la Comunicación Interna, pero aún no hallamos un alto grado de formalización de programas de Comunicación Interna. Persiste en la estructura mental de la alta dirección de las empresas, la necesidad de explicar adecuadamente la toma de decisiones a los públicos externos (clientes, inversores, autoridades, periodistas, etc.) antes que a los internos.

Definir la Comunicación Interna es hablar de sus finalidades, y éstas son:

- Influir sobre los empleados para que quieran aportar lo mejor de su profesionalidad al logro de los objetivos de la organización, se sientan orgullosos y valoren el trabajo realizado.

- Facilitar el diálogo entre la empre3a y sus empleados, ayudando a poner de manifiesto y a ampliar la convergencia entre necesidades y expectativas de ambos.

- Transmitir ilusión y compromiso y ayudar a hacer evolucionar la cultura corporativa.

- Difundir un estilo de gestión que aporte credibilidad y confianza al proyecto empresarial.

La empresa Bimbo, falló al mostrar un estilo de gestión que no favorecía ni la credibilidad ni la confianza de los empleados en su alta dirección. El caso fue el siguiente: Un técnico de la factoría de Granollers (Barcelona) envió por correo electrónico a varios compañeros, desde su domicilio particular fuera de horas de trabajo, un chiste sobre la empresa que ridiculizaba los sueldos. Las carcajadas fueron circulando hasta la dirección. La empresa aplicó el despido disciplinario "por pérdida de confianza y sentimiento de ofensa" ante el contenido del mensaje.

La medida desató una convocatoria de huelga general de 24 horas por parte del comité, con el apoyo de todos los empleados y sindicatos. Además, el trabajador demandó a la empresa por considerar "nulo" el despido y "desproporcionada" la sanción.

La empresa que popularizó el lema "lo más tierno" en los años 90 (excelente campaña donde se combinaron objetivos de Comunicación Interna con objetivos de Comunicación Externa), cometió el error de olvidar el factor humano que debe tener en consideración toda empresa. La lección está clara: antes de que una empresa adopte una decisión que puede afectar al público, su dirección debe tratar de contemplar la acción desde la perspectiva de un tercero. La dirección empresarial debe ser receptiva al modo en que sus empleados perciben las acciones emprendidas. Si los empleados consideran que la dirección les trata de modo injusto, su rendimiento se ve afectado y se desarrollan tensiones internas y el absentismo se dispara.

La Comunicación Interna es herramienta fundamental en la gestión de las empresas, su objetivo es obtener la máxima rentabilidad de todas las personas que la conforman y, para ello, se ha de conseguir transmitir una imagen positiva y un clima adecuado. El capital humano, al igual q5e el "otro" capital, debiera figurar en el balance de las empresas, pues no 
tiene el mismo valor una empresa en la que sus empleados están contentos de pertenecer a la organización y existe un verdadero espíritu de cultura corporativa, que una empresa donde esto no existe. En la empresa donde se ha cuidado el talento, hay más valor. «Mientras la experiencia técnica (capital intelectual) de una empresa es clave para su éxito, son las emociones de las personas las que transforman el valor intelectual en capital financiero " ${ }^{4}$. De ello podemos deducir que, para que las personas trabajen de manera efectiva y rentable, es necesario que se identifiquen y comprometan con las metas de la organización. Para PriceWaterhouse Coopers, una de las consultoras de comunicación más importantes a nivel mundial, los dos elementos más motivadores para la innovación son «la pasión y la confianza».

\section{Cuando llegan conflictos}

«Los ejecutivos de las organizaciones preparadas ante la crisis consideran sus empresas no solamente sistemas productivos sino también potencialmente destructivos. Los ejecutivos de estas empresas discuten puntos relacionados con el éxito, el liderazgo, el crecimiento y la excelencia, pero también consideran y discut\%n puntos que tienen que ver con potenciales fracasos, averías, deterioros y muertes. Han desarrollado la capacidad de imaginar lo peor, lo impensable, lo indecible, como una manera de hacer todo lo posible para evita tales sucesos. En contra de lo que convencionalmente se cree, la crisis no sucede y basta. Este cambio de filosofía corporativa tiene un importante impacto en las definiciones de excelencia corporativa» 5 .

La fuerza laboral de la empresa es una de las claves de su éxito en momentos de "paz" y de "guerra", y una Comunicación Interna eficaz y eficiente es imprescindible en una empresa moderna con proyección de futuro, es por ello que la empresa ha de concederle la atención necesaria a la estrategia de Comunicación Interna. Pero en ocasiones se produce una alarmante diferencia en la percepción del clima laboral por parte de la alta dirección y los empleados, lo que acarrea consecuencias potencialmente peligrosas. Estas situaciones se dan, sobre todo, en momentos de crisis en la empresa ya sean originados en su seno o provenientes del exterior.

Una crisis debe entenderse como cualquier disfunción que atenta contra el proyecto empresarial y altera el normal desenvolvimiento de la actividad corporativa: un conflicto laboral, un fallo de producción, un problema financiero, un accidente, cualquier tema que suscita el interés de los medios de comunicación, problema de distribución, de atención al cliente, la actuación de la empresa cuestionada, etc., cualquier asunto que afecte a la imagen y a la reputación corporativa.

El primer escudo ante una crisis es una buena reputación corporativa. Una buena imagen consolidada en el tiempo y ante sus públicos prioritarios, y entre ellos el público interno.

Es en estos momentos de conflicto donde encontraremos en la Comunicación Interna dos grandes ventajas:

\footnotetext{
${ }^{4}$ Cervera, L.A. Comunicación total. ESIC. Madrid, 2004. Pág. 278.

${ }^{5}$ Mitroff, I.
} 
1. La preventiva, porque una fluida y correcta Comunicación Interna nos ha ayudado a construir el clima interno positivo, nos ha servido para que el empleado confíe en la empresa y que se sienta identificado con los problemas de la empresa, sienta que también le afectan a él y eso le convierte en sujeto importante para la solución en momentos duros.

Otro de los puntos a tener en cuenta es que mantener una comunicación directa entre todos los miembros de la empresa es altamente positivo. Si jefes y empleados hablan abiertamente de cómo están trabajando juntos, se reduce el potencial de conflictos y se establecen relaciones laborales más fuertes y efectivas.

2. La operativa. Tener una Comunicación Interna bien gestionada nos ayudará, en el momento de crisis, a ser rápido, a tener los efectivos materiales y humanos necesarios en el momento justo, a evitar los momentos de ansiedad y nerviosismos con personas acostumbradas a manejarse con la Comunicación Interna y a manejar las herramientas para encontrar soluciones. Esto es iuy importante en la gestión de la comunicación de crisis, puesto que, una mala acción, una deficiente gestión de comunicación, y la empresa se juega la superación de la crisis.

Por otra parte, si se ofrece una información eficaz y fiable se cierra el camino a bulos y especulaciones, y sitúa la negociación en ambientes racionales, de los cuales, obviamente es más fácil esperar respuestas lógicas, por el contrario, una disminución en la calidad de la información, añade dificultades al ya difícil terreno de la negociación colectiva, debiendo llegarse a la información por canales paralelos, poco eficientes y peligrosos.

Se pueden identificar ocho resultados potenciales fruto de una mala dirección de comunicación en situaciones de crisis ${ }^{6}$ :

- pérdida de confianza en la organización y, sobre todo en la dirección, por parte de los inversores, clientes y trabajadores. Se daña la imagen y reputación corporativa.

- deterioro de la moral de los trabajadores. Falta de motivación que puede conducir a problemas laborales.

- disminución de precios de stock y relaciones tensas con los inversores.

- pérdida de tiempo y de fuerzas por parte de la dirección al estar polarizada únicamente en asuntos relacionados con la crisis, en lugar de resolver otras actividades organizacionales importantes.

- aumento de escrutinio de asuntos organizacionales por parte de agencias políticas externas, conduciendo a una regulación gubernamental excesiva y a medidas punitivas.

- $\quad$ litigios costosos en tiempo y en dinero.

- amenazas para la autonomía organizacional, como la reorganización voluntaria o la bancarrota

- relaciones tensas con la comunidad y en definitiva con el entorno.

Como hemos visto en el punto 1 y 2 de la relación anterior, en los momentos de conflicto, consecuencias seguras serán la falta de confianza de los empleados y la pérdida de motivación, por lo que, a la ya de por sí complicada crisis, la empresa puede sumar más

\footnotetext{
${ }^{6}$ Del Pozo, M. Cultura empresarial y Comunicación Interna. Fragua. Madrid, 1997. Pág. 27.
} 
dificultades. Lo cierto es que la crisis pueda hacer aflorar otro tipo de crisis dentro de la empresa, la realidad está llena de estos casos, pero también es cierto que muchaw veces ante una crisis bien resuelta a todos los niveles, y esto incluye forzosamente el nivel comunicativo, la imagen de la empresa puede salir muy reforzada.

En definitiva, una correcta actuación en los conflictos gracias a que la empresa gestiona profesional y correctamente su comunicación de crisis servirá para:

Evitar la metástasis. Una acción preventiva de la propagación de la crisis en el interior de la empresa es tener una Comunicación Interna bien gestionada. Todo será más fácil y el cambió será mejor asumido por todos. Los empleados, que comparten la visión de la empresa y la apoyan, sentirán como suya la crisis y ayudarán a superarla. Servirá para aliviar las tensiones cuando se comparte información, compromisos y participación.

Silenciar radiomacuto. Uno de los peores problemas con los que se encuentra la empresa ante una situación considerada de crisis es la aparición de los rumores. En estos momentos la organización sufrirá más de lo necesario a causa de este fenómeno, ya que los rumores crean incertidumbre, la incertidumbre produce temor y el temor no permite trabajar de forma eficiente.

Aportar transparencia. En los momentos de crisis ante el conflicto, el máximo enemigo de una empresa es el silencio, ya que supone la aceptación de culpa. Su solución pasa por la transparencia, ya que aporta credibilidad a los contenidos de los mensajes transmitidos. La opacidad informativa es contraproducente, sobre todo si más adelante se demuestra que ha habido ocultación de datos.

Buscar soluciones. La Comunicación Interna y el diálogo son las herramientas para lograr que los conflictos laborales se reconduzcan hacia un cambio positivo en las organizaciones. Hay que evitar los nocivos efectos secundarios de no actuar bien a nivel comunicativo, después de todo no hay que olvidar que hay emociones de por medio, y se trata de humanizar la actuación de la empresa. Nada hay más humano que la comunicación bien entendida.

\section{Manos a la obra}

Las premisas a la hora de actuar será poner en marcha el plan de comunicación de crisis que se tiene definido en la empresa sin olvidar que se debe:

- Anticipar la información todo lo que sea posible, antes de que aparezcan problemas.

- Completar la información y ampliarla cuando se produzcan aconte-cimientos.

- Estar prevenidos para informar con celeridad.

- No presuponer que los empleados estarán informados por otros conductos.

\section{La tecnología aliada}

Una Intranet, un portal del empleado, sería una aportación muy importante a la hora de gestionar el conflicto, como medio accesible, rápido y versátil. Donde se puede personalizar la información interna. La e-comunicación para empleados serviría para dar estructura, significado y orden a toda la situación de crisis originada, donde el público interno se informa y se forja como grupo al encontrar el canal idóneo para su información y el cauce de sus inquietudes, que en esos momentos pueden ser muchas. La e-comunicación dentro de la empresa es una herramienta poderosa que posee grandes virtudes como la versatilidad, la 
inmediatez, la globalidad, etc., pero entre todas ellas destaca una: la interactividad, que se puede manejar en beneficio de la empresa.

\section{Es necesaria la gestión profesional de la comunicación corporativa}

Para llevar a cabo todo esto es necesaria la existencia de la figura del Director de comunicación dentro de la empresa. La figura de responsable de la comunicación corporativa tal y como la define Joan Costa, el estratega ${ }^{7}$. El director de Comunicación no es un mero gestor de las herramientas de comunicación sino que es el que decide desde una visión panorámica tanto por sus conocimientos y capacidad como por su situación. Esto ofrece una visión diferente a lo que debe ser un Director de Comunicación, como dice Costa «más importante que "saber hacer bien" una cosa, es saber qué cosa es necesaria hacer en cada momento».

He aquí una figura que no es ni solamente el planificador ni el director de la ejecución, sino mucho más que todo eso. Es el responsable de la comunicación corporativa. Estratega más que director. Conceptualizador más que técnico. Planificador y supervisor permanente, más que ejecutante. Y para todo esto no sirve cualquiera, sino un profesional de la comunicación, formado en la universidad en la disciplina de Publicidad y Relaciones Públicas.

\section{Conclusión}

La fuerza laboral de la empresa es una de las claves de su éxito, y la Comunicación Interna eficaz y eficiente es imprescindible en una empresa moderna con proyección de futuro, es por ello que la empresa ha de concederle la atención necesaria a la estrategia de Comunicación Interna. Una política preactiva basada en la buena imagen, predispone favorablemente a los públicos. Una buena Comunicación Interna fragua fortalezas en el seno de la empresa y dota de un escudo de imagen a nivel externo ${ }^{8}$. Esto permite partir con ventaja.

En definitiva, gestionar profesionalmente la Comunicación Interna en la empresa sirve para detectar y solucionar los conflictos, y reporta ventajas tanto para los empleados como para la empresa al favorecer el clima interno. En el plano interpersonal permite establecer relaciones más sólidas, aumentar el respeto por la empresa y sus componentes, y potenciar el desarrollo personal. Mientras que para la compañía supone mejorar la eficacia del trabajo y las acciones emprendidas, a la vez, se fomenta la creatividad, se logra una sinergia que se traduce en mayor desarrollo y se favorece el crecimiento de beneficios. $Y$ gracias a todo este correcto trabajo, en momentos de conflicto, servirá para amortiguar el desacuerdo, y llevar a una pronta y mejor solución.

\section{Bibliografía}

DIRCOM. Expectativas, prácticas y resultados de la Comunicación Interna en empresas e instituciones españolas. Dircom. Madrid, 2003.

GRUNIG, J.E. y HUNT, T. Dirección de Relaciones Públicas. Gestión 2000. Barcelona, 2003.

Costa, J. Comunicación corporativa y revolución de los servicios. Ediciones Ciencias Sociales. Madrid, 1995.

Lacasa, A.S. Gestión de la comunicación empresarial. Gestión 2000. Barcelona, 2004. 
LA BUENA GESTIÓN DE LA COMUNICACIÓN INTERNA UNA VENTAJA A FAVOR DE LAS EMPRESAS ANTE LOS CONFLICTOS

CERVERA, L.A. Comunicación total. ESIC. Madrid, 2004

DEL POZO, M. Cultura empresarial y Comunicación Interna. Fragua. Madrid, 1997.

LACASA, A.S. Gestión de la comunicación empresarial. Gestión 2000. Barcelona, 2004.

COSTA, J. Comunicación corporativa y revolución de los servicios. Ediciones Ciencias Sociales. Madrid, 1995. 